\title{
A MODEL OF THE MAMMALIAN RETINA AND THE VISUAL CORTEX. DISORDERS OF VISION.
}

\author{
JA. Martín-Pereda, C. Sánchez-Guillén and A. González-Marcos \\ E.T.S.I. Telecomunicación. Universidad Politécnica de Madrid \\ Ciudad Universitaria, 28040 - Madrid, Spain \\ e-mail: JAMP@TFO.UPMES
}

\begin{abstract}
A model of the mammalian retina and the behaviour of the first layers in the visual cortex is reported. The building blocks are optically programmable logic cells. A model of the retina, similar to the one reported by Dowling is presented. From the model to the visual cortex obtained, some type of symmetries and asymmetries are possible to be detected.
\end{abstract}

\section{INTRODUCTION}

One of the most challenging problems that must be solved by any theoretical model purporting to explain the competence of the human brain for relational tasks is the one related with the analysis and representation of the internal structure in an extended spatial layout of multiple objects. In this way, some of the problems are related with specific aims as how can we extract and represent spatial relationships among objects and how can we represent the movement of a selected object

The main objective of this paper is the study of some plausible brain structures that can provide answers in these problems. Moreover, in order to achieve a more concrete knowledge, our study will be focused on the response of the retinal layers for optical information processing and how this information can be processed in the first cortex layers.

\section{NEUROPHYSIOLOGICAL BASIS OF THE MODEL}

According to a well-known idea [1], the retina is the most approachable part of the brain. It is because that, the visual system is very attractive to be modelled: it is easily stimulated with light and it is naturally divided between eye and brain. A model of the mammalian retina, previously reported by us [2]-[3], will be employed here as the first step to provide information to the visual cortex. Our model was based on the one proposed by Dowling [1]. It is composed by two photoreceptors, one horizontal cell, two bipolars, one amacrine and three ganglion cells. The way these cells have been simulated is with the previously reported logic cells, OPLs, [4]-[5], employed as basic units for optical computing architectures. Their basic structure is shown in Fig. 1. A brief description on their method of operation, as well as the way they have been implemented, can be seen in [4]-[5]. The main circuit is composed by two optical devices, $\mathrm{P}$ and $\mathrm{Q}$, with a non-linear behaviour. The output of each one of them corresponds to the two final outputs, $\mathrm{O}_{1}$ y $\mathrm{O}_{2}$, of the cell. The possible inputs to the circuit are four. Two of them are for the input data, $\mathrm{I}_{1} \mathrm{e}_{2}$, and the other two, $g$ and $h$, for control signals. The way these four inputs are distributed inside the circuit it is also represented in Figure 1. The corresponding inputs to the non-linear devices are based on these signals plus some other coming from inside the own cell. The characteristics of the non-

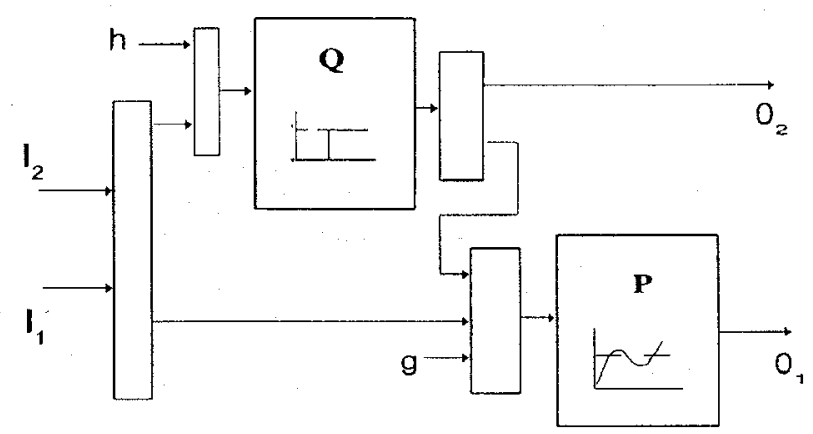

Fig. 1.- Optically programmahle logic cell.

linear devices are also shown in Fig. 1. The functions that have being obtained from this structure are fourteen pairs of logic functions. Details are given in [4]-[5]. These cells have been the main units to construct a model of the mammalian retina, according to the Dowling's one.

Control signals imposed to photoreceptor cells make them to operate with NAND functions. Same function is performed by the horizontal cell $\mathrm{H}$. Bipolar cells operate as AND or NAND functions, according to the control signals imposed by the previous layer. Another type of function is performed by the amacrine cell. It gives a periodic signal, with period and pulse length depending on the characteristics of the signals coming from the bipolar cells. The composite signals from bipolar and amacrine cells arrive to the ganglion cells from where the final signal is obtained. In the studied case, three are the outputs. The first one is a train of light pulses being its length the same one as the input flash. The third one is always a sequence of short pulses; only when it was light at the input, pulses disappear. Finally, the second one gives a very short train of pulses at the beginning and at the end of the initial flash. Some more details are given in [2]-[3] and will be presented. 


\section{RESULTS CONCERNING THE LOWER VISUAL CORTEX}

A further step has been undertaken based on the above obtained results. Moreover, this new work is the first step towards a more complex simulation In this case, a simple linear configuration has been adopted for the first layer of our model. It corresponds to the possible signals coming out from ganglion cells. These signals are the result of the corresponding input data impinging onto the receptors. They give information about time length of the input light pulse and, in our linear case, number and type of receptors with incoming light. This number has a close correspondence with the spatial length of the signal.

These signals are compared at a second layer, in the way shown in Fig. 2. Boxes correspond to the previously mentioned OPL cells. Output data are compared at the third and following layers in the same way than before. The process keeps going on until it reaches layer 6 where there is just one single output.

The possibilities from this architecture are as many as possible logic functions are able to perform the OPLs. This situation gives the possibility to implement any type of synapses between neurons, both excitatory and inhibitory, as well as oscillatory behaviours. The reported architecture is

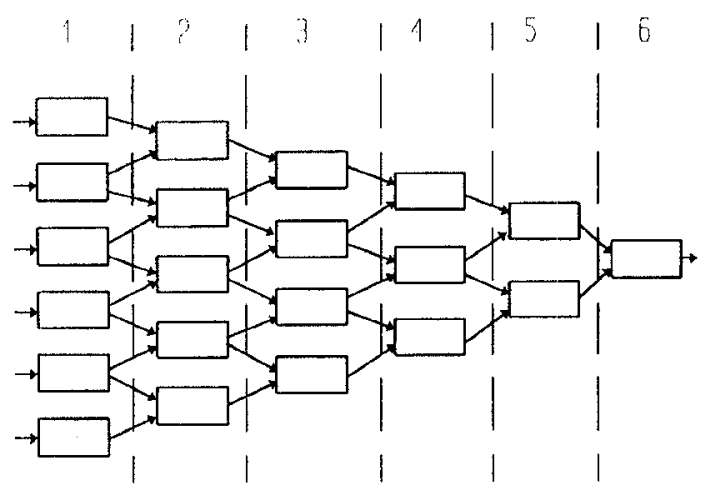

Fig. 2.- Hierarchical anrangement for the visual cortex.

just a two dimensional configuration without lateral branches corresponding to other possible functions. Because the whole information corresponding to the input image is transferred to a train of pulses in each type of ganglion cells, in order to process such an information, a different type of architecture will have to be implemented for each one of the input data (for example, colours, shapes, motion and edges)

We have considered in this occasion just one of the two possible outputs from the OPLs. Moreover, just one type of logic function was adopted, namely, XOR. With these boundary conditions, our structure is able to recognize asymmetries in the input signals. If some type of symmetry is present on the signal impinging onto the first layer of cells, a "l" will be obtained at the output. On the contrary, asymmetries will give rise to a " 0 " at the final cell. Some other features could be implemented with similar configurations.

\section{DISORDERS OF VISION}

As it was shown [5], if a certain feedback is added to the OPL, when external or internal delays are varied according to certain rules, a chaotic process could be obtained. This new state may appear from many different sources. In the case of an optoelectronic simulation, a variation in the nonlinear device parameter values can be the origin of these changes. For example, they may be originated from a certain increase in temperature or a bias fluctuation.

In the reported case, the output from layer 6 could vary from " 1 " to " 0 ", or viceversa, in chaotic way. Because this signal gives information about the external world, no true information is obtained. Hence, a malfunction at a certain layer of the cortex would give rise to disorders of vision. A computer simulation has been developed.

\section{V.- CONCLUSIONS}

We have obtained, with the reported architecture, a possible way to simulate the first layers of the visual cortex. The present configuration is able to process almost any type of information acting on the retinal receptor. The final output is a bit of information that could be considered as a bit of knowledge. Some other possibilities will be reported as well as a possible way to explain vision disorders.

\section{IV.- ACKNOWLEDGMENTS} grant TIC95-0118.

This work was partly supported by the CICYT,

\section{V.- REFERENCES}

[1] Dowling, JE "The Retina. An Approachable part of the Brain". Harvard Univ. Press. London.. 1987.

[2] Martín-Pereda, IA. \& A. González-Marcos. "An Approach to Visual Cortex Operation: Optical Neuron Model". CLFOFfumope-EQFC94. Amsterdam. 1994.

[3] González-Marcos, A. \& J.A. Martín-Pereda "A Model of Amacrine and Ganglion Cells for Optical Information Processing". LEOS94. Boston. 1994.

[4] Martín-Pereda, JA. \& A. González-Marcos. "Optical Programmable Processing Element using Optical Fibers". IEEE Lasers and Electro-Optics Society, LEOS'92. Boston, 15-20 November, 1992.

[5] González-Marcos, A. \& JA. Martín-Pereda, "Digital Chaotic Output from an Optically-Processing Element". Optical Engineening, 35, pp. 525-535, 1996. 